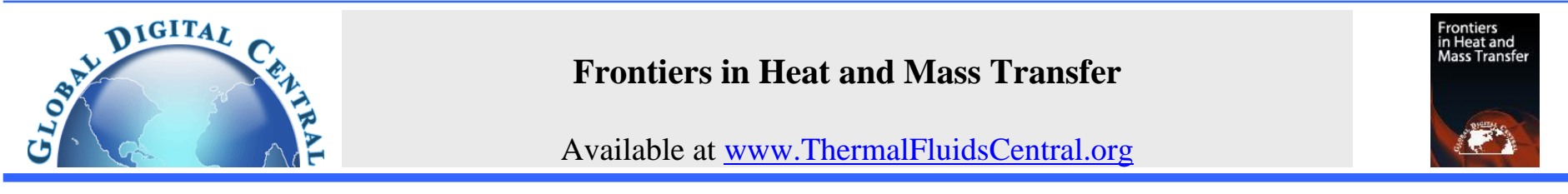

\title{
EFFECTS OF CHEMICAL REACTION AND THERMAL RADIATION ON HEAT GENERATED STRETCHING SHEET IN A COUPLE STRESS FLUID FLOW
}

\author{
G. Nagaraju ${ }^{\mathrm{a}, \dagger}$, Anjanna Matta $^{\mathrm{b}}$, K. Kaladhar ${ }^{\mathrm{c}}$ \\ a Department of Mathematics, GITAM University, Hyderabad campus, Rudraram(V), Medak dist., Telangana, India \\ ${ }^{\mathrm{b}}$ Department of Mathematics, Indian Institute of Technology Hyderabad, Kandi, Telangana-502285, India \\ ${ }^{\mathrm{c}}$ Department of Mathematics, National Institute of Technology Puducherry, Karaikal-609605, India
}

\begin{abstract}
A theoretical steady of two-dimensional and MHD couple stress fluid flow over a linearly stretching sheet is investigated with the effects of thermal radiation, internal heat generation and homogeneous chemical reaction of first order. The governing equations of continuity, momentum, energy and diffusion for this boundary layer flow are transformed into one set of coupled non-linear ordinary differential equations using the local similarity transformation and are then solved using the fourth-order Runge-Kutta method along with the shooting technique. The effects of the couple stress parameter $(S)$, Magnetic parameter $(M)$ and chemical reaction parameter $(C r)$ are presented through the graphical illustrations. It has been found from the results that the temperature of the couple stress fluid is enhanced due to increases in $\operatorname{Pr}, E c, M$ and $Q$ but decreased with $R$ and $S$. Increase in the couple stress parameter $(S)$ enhances the flow velocity but enhance in the magnetic field $(M)$ reduces the flow velocity.
\end{abstract}

Keywords: viscous dissipation, couple stress fluid, heat generation, stretching sheet, Radiation.

\section{INTRODUCTION}

The study of chemical reaction and magnetic field has significant role in the areas of industrial and engineering sciences. The major practical applications can be found in petroleum industry, chemical processing and cooling of nuclear reactors etc.,. In view of this practical problems, many researchers have reported their results with influence of the chemical reactions on double diffusive convection applications. The homogeneous first order chemical reaction, MHD effects on viscous flow past an inclined flat plate with mixed convection has been presented by Alam et al. (2008). Rahman and Al-Lawatia (2010) examined the effect of chemical reaction with higher order and variable concentration of the reactant on the flow of micropolar fluid over a non-linear permeable stretching sheet. Ferdows and M. Al-Mdallal (2013) analyzed the impact of the order of chemical reaction over a linearly stretching sheet in a viscous fluid flow. Shaw et al. (2013) examined that the influence of heterogeneous and homogeneous reaction on the micropolar fluid flow past a porous stretching/shrinking sheet with suction. Recently, Patil and Chamkha (2013) reported on mixed convective flow of polar fluid with the effects of chemical reaction and internal heat generation.

In recent years, considerable attention has been devoted to radiative double diffusive convection due to importance in manufacturing industry such as design of furnace, glass production, power plants, casting, levitation, gas turbines, energy utilization, satellite and space vehicles etc.,. In view of applications, Patil and Kulkarni (2008) presented the natural convection flow of polar fluid with heat generation and chemical reaction effects in porous medium. Kameswaran et al. (2012) analyzed the homogeneous-heterogeneous reactions in micropolar fluid flow from a permeable stretching or shrinking sheet in a porous medium. Hussain et al. (2013) investigated the unsteady micropolar fluid flow over a permeable stretching sheet with the effect of thermal radiation. Govardhan et al. (2015) studied boundary layer analysis for magnetohydrodyamics (MHD) and radiation effects on mixed convection, unsteady flow of incompressible micropolar fluid over a stretching sheet.

Many fluids in technical processes exhibit non- Newtonian behavior since the conventional Newtonian fluids are not specifically explains the properties of real fluids. Among all such non- Newtonian fluids, Stokes (1966) introduced the couple stress fluids, which have different features such as non-symmetric stress tensor, body couples and couple stresses. These fluids are able to describe various types of blood, suspension fluids, lubricants etc. Soundalgekar (1971) reported on solute dispersion effect in a channel with couple stress fluid. Chang-Jian and Chen (2008) examined the non-linear suspension of flexible rotor in presence of couple stress fluid film bearings. The effects of thermal convection over a nonlinear stretching sheet have been presented by Khan et al. (2013). Hayat et al. (2013) discussed the nature of couple stress fluid flow with effect of melting heat transfer over a stretching sheet. Turkyilmazoglu (2014) has been examined the MHD couple stress fluid flows over a shrinking

${ }^{\dagger}$ Corresponding author. Email: naganitw@gmail.com 
permeable or continuously stretching sheet. Even recently, Khan et al. (2014) presented the two-dimensional MHD couple stress fluid flow over a non-linear stretching sheet with the influence of chemical reaction.

The main focus of this research article is to analyze the effects of various governing flow parameters on couple stress fluid over a linearly stretching sheet with thermal radiation, first order chemical reaction and internal heat generation effects. The above literature clearly shows that the physical significance of the effects of the present effects considered in non- Newtonian fluids along a stretching sheet. The survey clearly shows that the combined effects on couple stress fluid on heat generated stretching sheet has not been presented else where. The governing coupled nonlinear equations are transformed to system of ordinary differential equations using the appropriate similarity transformations. Shooting method with fourth order Runge - Kutta method has been used to solve the final system of equations. Effects of various pertinent parameters on the flow field have been presented through plots.

\section{MATHEMATICAL ANALYSIS}

Consider an electrically conducting incompressible couple stress fluid flow over a internal heat generated linearly stretching sheet with the effects of radiation and chemical reaction. In addition, a constant magnetic field is applied normal to the sheet. The temperature and concentration at the plate are notated as $T$ and $C$, respectively. The ambient temperature and concentration of the stretching sheet are $T_{\infty}$ and $C_{\infty}$ respectively. With the above presumptions, the governing equations for the incompressible steady flow of couple stress fluid with MHD approximations are

$$
\begin{gathered}
\frac{\partial u}{\partial x}+\frac{\partial v}{\partial y}=0 \\
u \frac{\partial u}{\partial x}+v \frac{\partial u}{\partial y}=\frac{\mu}{\rho} \frac{\partial^{2} u}{\partial y^{2}}-\frac{\eta_{0}}{\rho} \frac{\partial^{4} u}{\partial y^{4}}-\frac{\sigma B_{0}^{2}}{\rho} u \\
u \frac{\partial T}{\partial x}+v \frac{\partial T}{\partial y}=\alpha \frac{\partial^{2} T}{\partial y^{2}}+\frac{\mu}{\rho c_{p}}\left(\frac{\partial u}{\partial y}\right)^{2}+\frac{\eta_{0}}{\rho c_{p}}\left(\frac{\partial^{2} u}{\partial y^{2}}\right)^{2} \\
-\frac{1}{\rho c_{p}} \frac{\partial q^{r}}{\partial y}+\frac{Q_{0}}{\rho c_{p}}\left(T-T_{\infty}\right), \\
u \frac{\partial C}{\partial x}+v \frac{\partial C}{\partial y}=D \frac{\partial^{2} C}{\partial y^{2}}-k_{1}\left(C-C_{\infty}\right),
\end{gathered}
$$

The velocities in the direction of $x$ and $y$ are $u$ and $v$ respectively, $c_{p}$ is the specific heat, $\rho$ is the density, $\mu$ is the coefficient of viscosity, $\eta_{0}$ is the additional viscosity coefficient which specifies the character of couple-stresses in the fluid, $B_{0}$ is the applied magnetic field, $\sigma$ is the electrical conductivity of the fluid, $D$ is the mass diffusion coefficient, $\alpha$ is the thermal conductivity of the fluid and $k_{1}$ is the chemical reaction parameter.

The subsequent boundary conditions are

$$
\begin{array}{r}
u=u_{w}(x)=c x, v=0, \frac{\partial^{2} u}{\partial y^{2}}=0, T=T_{w}, C=C_{w} \quad \text { at } \quad y=0 \\
u \rightarrow 0, \frac{\partial u}{\partial y} \rightarrow 0, T \rightarrow T_{\infty}, C \rightarrow C_{\infty} \quad \text { as } \quad y \rightarrow 0
\end{array}
$$

The radiative heat flux $q^{r}$ by using the Rosseland approximation is

$$
q^{r}=-\frac{4 \sigma_{s}}{3 k_{e}} \frac{\partial T^{4}}{\partial y},
$$

where $k_{e}$ and $\sigma_{s}$ are the mean absorption coefficient and Stefan-Boltzmann constant respectively. Eqn. (6) can be linearized by taking Taylor series expansion of $T^{4}$ about $T_{\infty}$ (higher order terms are neglected) when the thermal differences within the flow are adequately small.

$$
T^{4} \cong 4 T T_{\infty}^{3}-3 T_{\infty}^{4}
$$

In view of (6) and (7), (3) reduces to

$$
\begin{aligned}
u \frac{\partial T}{\partial x}+v \frac{\partial T}{\partial y}=\alpha & (1+R) \frac{\partial^{2} T}{\partial y^{2}}+\frac{\mu}{\rho c_{p}}\left(\frac{\partial u}{\partial y}\right)^{2}+\frac{\eta_{0}}{\rho c_{p}}\left(\frac{\partial^{2} u}{\partial y^{2}}\right)^{2} \\
& +\frac{Q_{0}}{\rho c_{p}}\left(T-T_{\infty}\right)
\end{aligned}
$$

where $\alpha=\frac{k}{\rho c_{p}}$ is the thermal diffusivity and $R=\frac{16 \sigma_{s} T_{\infty}^{3}}{3 k k_{e}}$ is the radiation parameter.

Introducing the following similarity transformations

$$
\left.\begin{array}{c}
u=c x f^{\prime}(\eta), \quad v=-\sqrt{c \nu} f(\eta), \eta=\sqrt{\frac{c}{\nu}} y, \quad \theta(\eta)=\frac{T-T_{\infty}}{T_{w}-T_{\infty}}, \\
\phi(\eta)=\frac{C-C_{\infty}}{C_{w}-C_{\infty}}
\end{array}\right\}
$$

in equations (2)-(8), we obtain the governing dimensionless equations as

$$
S f^{v}+\left(f^{\prime}\right)^{2}-f f^{\prime \prime}-f^{\prime \prime \prime}+M f^{\prime}=0,
$$

$$
\begin{gathered}
\left(\frac{1+R}{P r}\right) \theta^{\prime \prime}+f \theta^{\prime}-f^{\prime} \theta+E c\left(f^{\prime \prime}\right)^{2}+S E c\left(f^{\prime \prime \prime}\right)^{2}+Q \theta=0, \\
\phi^{\prime \prime}+S c\left(f \phi^{\prime}-f^{\prime} \phi-C r \phi\right)=0
\end{gathered}
$$

with

$$
\left.\begin{array}{c}
f(0)=0, \quad f^{\prime}(0)=1, \quad f^{\prime \prime \prime}(0)=0, \quad \theta(0)=1, \quad \phi(0)=1 \\
f^{\prime}(\infty)=0, \quad f^{\prime \prime}(\infty)=0, \quad \theta(\infty)=0, \quad \phi(\infty)=0
\end{array}\right\}
$$

Here $S=\frac{c \eta_{0}}{\rho \nu^{2}}$ is the couple stress parameter, $M=\frac{\sigma B_{0}^{2}}{c \rho}$ is the magnetic interaction strength parameter, $\operatorname{Pr}=\frac{\mu c_{p}}{k}$ is the prandtl number, $Q=$ $\frac{Q_{0}}{\rho c c_{p}}$ is the heat generation parameter, $E c=\frac{c^{2} x^{2}}{c_{p}\left(T_{w}-T_{\infty}\right)}$ is the Eckert number and $C r=\frac{k_{1}}{c}$ is the chemical reaction parameter.

In absence of the couple stress parameter $S$, the fluid is just the classical non polar regular and Newtonian. The major interest on physical parameters are skin- friction parameter $C_{f}$, local Nusselt number $N u_{x}$ and the local Sherwood number $S h_{x}$ which indicate physically the wall shear stress, the rate of heat transfer and the local surface mass flux respectively.

The wall shear stress $\tau_{w}$ is given by

$$
\tau_{w}=\left(\mu \frac{\partial u}{\partial y}-\eta_{0} \frac{\partial^{3} u}{\partial y^{3}}\right)_{y=0}=\mu c x \sqrt{\frac{c}{\nu}}\left(f^{\prime \prime}-\frac{c \eta_{0} f^{\prime \prime \prime \prime}}{\mu \nu}\right)_{y=0} .
$$

The dimensionless skin friction coefficient which impulsively started at the sheet is

$$
C_{f}=\frac{\tau_{w}}{\rho u_{w}^{2}}=C_{f} \operatorname{Re}_{x}^{\frac{1}{2}}=f^{\prime \prime}(0)-S f^{\prime \prime \prime \prime}(0) .
$$

The heat flux $q_{w}$ and mass flux $q_{m}$ are given by

$$
\begin{gathered}
q_{w}=-k\left(\frac{\partial T}{\partial y}\right)_{y=0}=-\theta^{\prime}(0)\left(T_{w}-T_{\infty}\right) \sqrt{\frac{c}{\nu}}, \\
q_{m}=-k\left(\frac{\partial C}{\partial y}\right)_{y=0}=-\phi^{\prime}(0)\left(C_{w}-C_{\infty}\right) \sqrt{\frac{c}{\nu}} .
\end{gathered}
$$

The non-dimensional shear stress, the Nusselt number and the Sherwood number are given by

$$
\begin{gathered}
N u_{x}=\frac{x q_{w}}{k\left(T_{w}-T_{\infty}\right)}=-\theta^{\prime}(0) R e_{x}^{\frac{1}{2}}, \\
S h_{x}=\frac{x q_{m}}{D\left(C_{w}-C_{\infty}\right)}=-\phi^{\prime}(0) R e_{x}^{\frac{1}{2}}
\end{gathered}
$$

where $R e_{x}=\frac{c x^{2}}{\nu}$ is the local Reynolds number. 


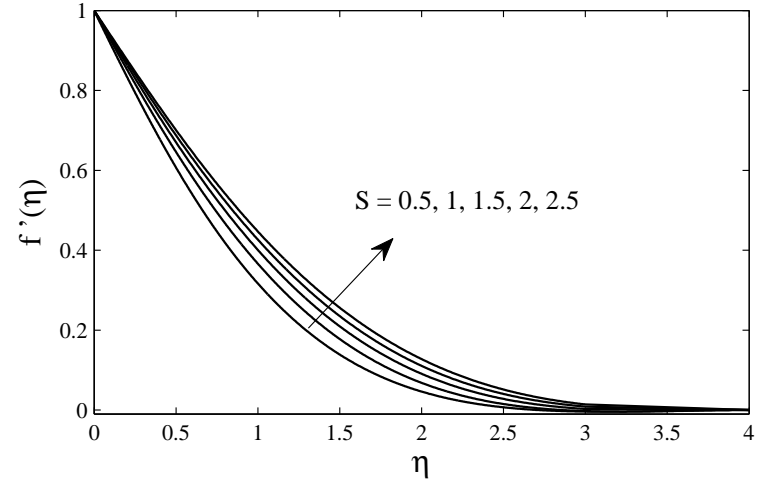

Fig. 1. Couple stress parameter effect on $f^{\prime}(\eta)$.

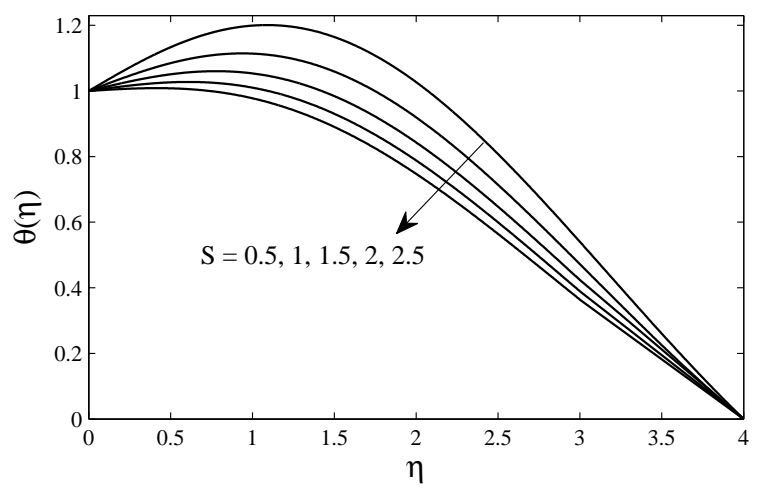

Fig. 2. Couple stress parameter effect on $\theta(\eta)$.

\section{RESULTS AND DISCUSSION}

The present study reports the influence of chemical reaction and various physical parameters on double diffusive convection in presence of couple stress fluid flow over a linear stretching sheet. The non-linear differential Eqs. (10)- (12) were solved numerically by using Shooting and forth order Runge-Kutta methods. Computations were carried out by fixing the parameters $M=5, R=0.5, S=2, P r=0.71, S c=0.7$, $C r=1, E c=0.05$ and $Q=1$ to analyze the effects of the emerging parameters. If any parameter used specifically, it is notated in that graph. The obtained results have been analyzed for various values of pertaining parameters.

Figs. 1 and 2 represents the effect of couple stress parameter on both velocity $f^{\prime}(\eta)$ and temperature $\theta(\eta)$ components. Fig. 1 shows that the velocity of the fluid increases as the couple stress parameter increases. It is noted from Fig. 2 that the dimensionless temperature of the fluid decreases with an increase in couple stress parameter. Thus, the presence of couple stresses in the fluid increases the velocity and decreases the temperature.

The velocity $\left(f^{\prime}(\eta)\right)$ and temperature $(\theta(\eta))$ profiles are displayed in Figs. 3 and 4 for various values of the $M$. It is clear from Fig. 3 that the velocity of the fluid decreases as the magnetic parameter $(M)$ increases. It is obvious that the nature of the transverse magnetic field normal to the flow direction has an affinity to create drag (nothing but Lorentz force), which leads to resist the flow. Therefore increase in magnetic parameter influences to decrease the flow velocity. Figure 4 shows that the temperature profile $(\theta(\eta))$ is enhanced with increase in the magnetic field. The energy dissipation is analyzed under the influence of various parameters $(\theta(\eta), \operatorname{Pr}, E c, Q$ and $R)$ on temperature and are shown in Figs. 5 - 8.

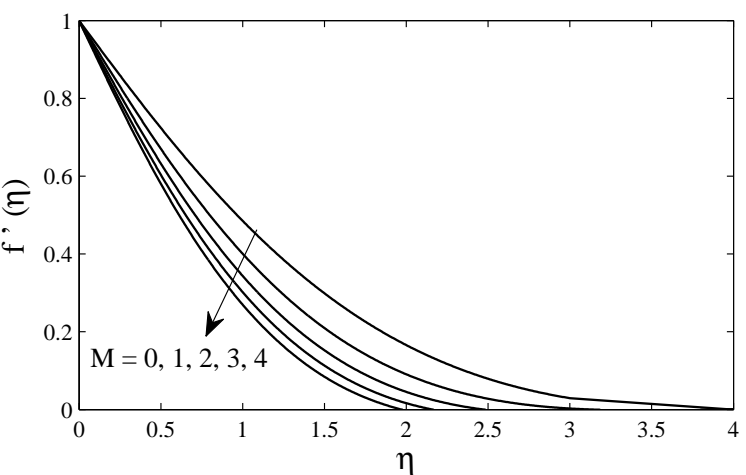

Fig. 3. Magnetic parameter effect on $f^{\prime}(\eta)$.

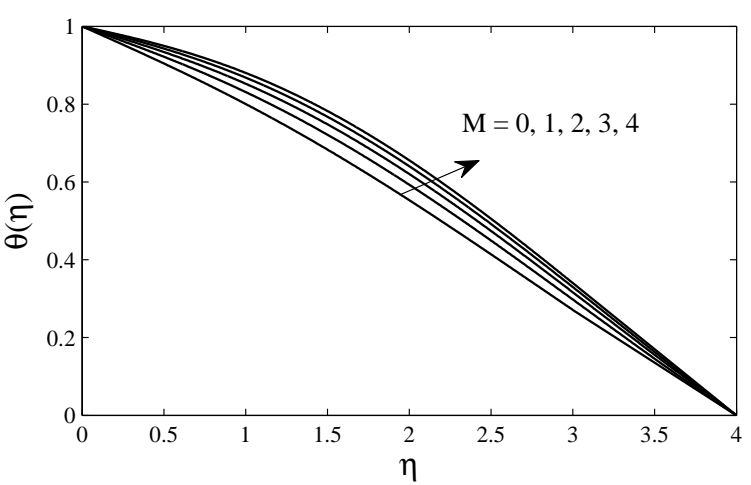

Fig. 4. Magnetic parameter effect on $\theta(\eta)$.

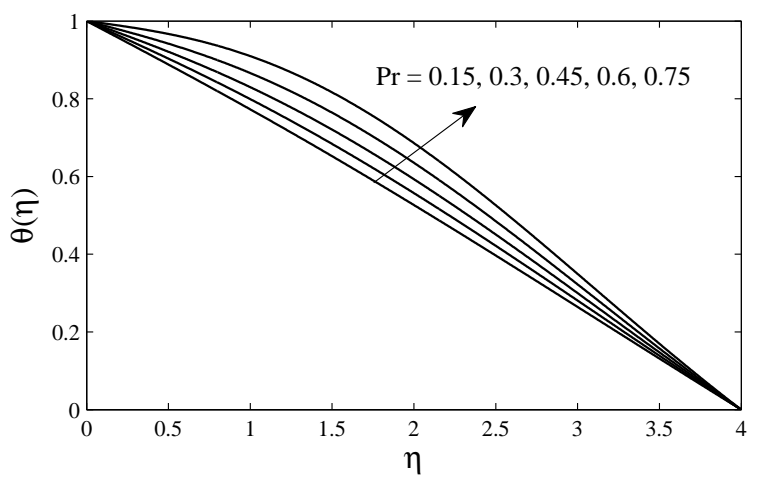

Fig. 5. Temperature profile $\theta(\eta)$ for various values of $P r$. 


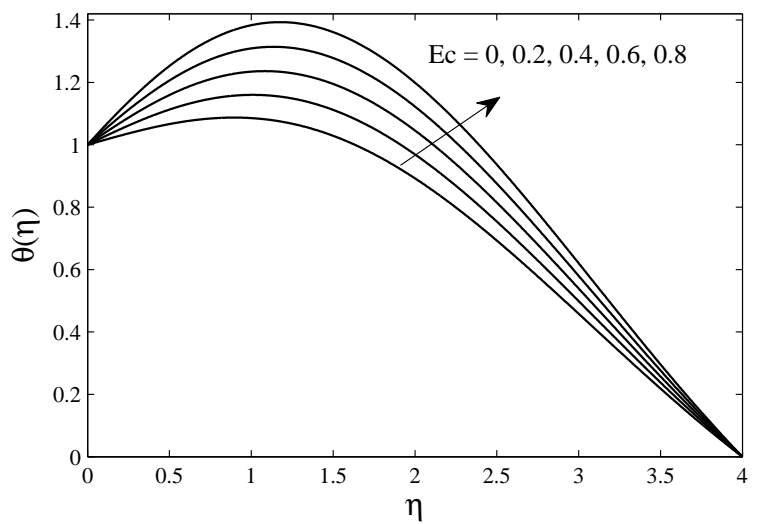

Fig. 6. Temperature profile $\theta(\eta)$ for various values of $E c$

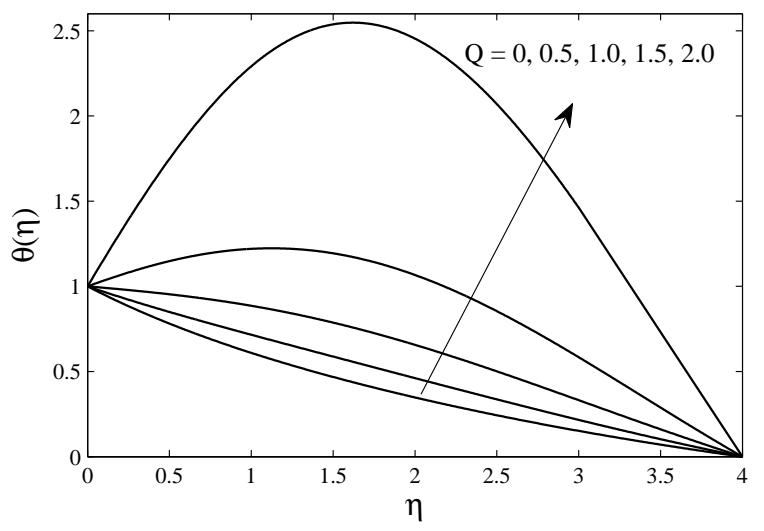

Fig. 7. Temperature profile $\theta(\eta)$ for various values of $Q$.

It is observed that the thermal boundary layer thickness increases with the increase of $\operatorname{Pr}, E c$ and $Q$ which leads to increase in the temperature profile $(\theta(\eta))$ and which can be seen in Figs. 5 - 7. The decreasing trend has been observed on the profiles of $\theta(\eta)$ as an increase in the Radiation parameter $R$ seen in Fig. 8. The increase in radiation parameter means that the release of heat energy from the flow region and so the fluid temperature decreases. It is also discovered that the boundary layer thickness has been decreased (see Figure 8). The effects of chemical reaction parameter $(\mathrm{Cr})$ and the Schmidt number $(\mathrm{Sc})$ on the concentration profile $\phi(\eta)$ are shown in Figs. 9 - 10. It can be seen from these figures that as an increase in the chemical reaction parameter $(\mathrm{Cr})$ and Schmidt number $(S c)$ results in the decrease in the concentration species $\phi(\eta)$.

The effects of couple stress fluid parameter and magnetic parameter on local skin friction, local Nusselt number and local Sherwood numbers are presented in Table 3. It is clear from the table that as $S$ increases the local skin friction coefficient decrease where as the Nusselt and Sherwood numbers are increases due to the thermal boundary layer thickness is decreased. It is also observed that all the physical parameters are decreases with an increase in the magnetic parameter. Since more magnetic effect suppress the flow rate. The present analysis shows that the flow field is appreciably influenced by the couple stress and magnetic parameters.

\section{CONCLUSION}

A detailed steady of two-dimensional laminar double diffusive convection over a stretching sheet has been investigated. The effect of first order

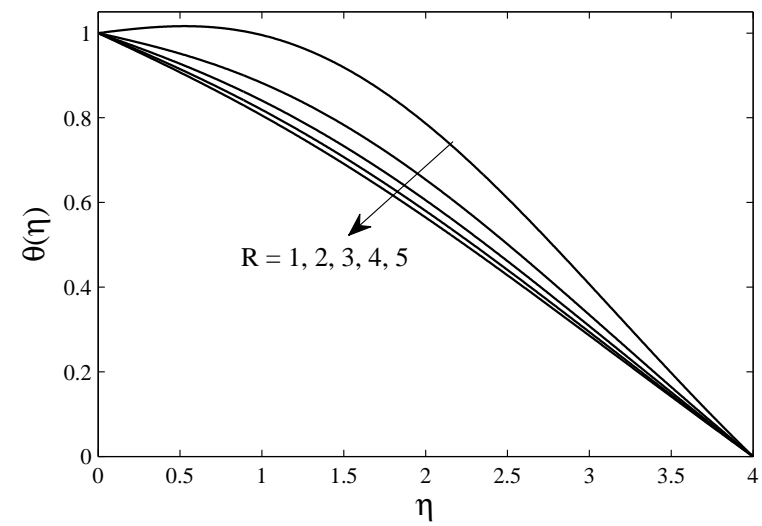

Fig. 8. Temperature profile $\theta(\eta)$ for various values of $R$

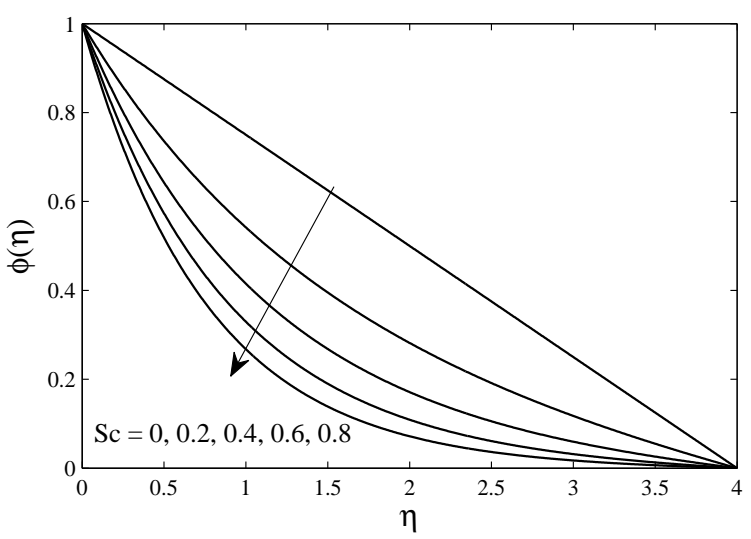

Fig. 9. Concentration profile $\theta(\eta)$ for various values of $S c$.

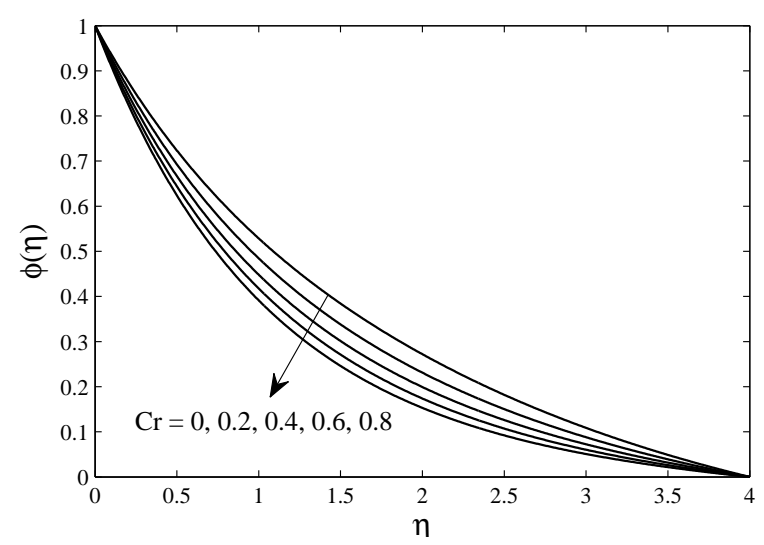

Fig. 10. Concentration profile $\theta(\eta)$ for various values of $C r$. 
Table 1: Variation of skin friction, Nusselt number and Sherwood number for various values of $S$ and $M$.

\begin{tabular}{|c|c|c|c|c|}
\hline$S$ & $M$ & $C_{f} R e_{x}^{1 / 2}$ & $N u_{x}$ & $S h_{x}$ \\
\hline 1 & 5 & -3.5085 & -0.6571 & 0.9692 \\
2 & 5 & -3.9256 & -0.4657 & 0.9871 \\
3 & 5 & -4.2250 & -0.3398 & 0.9982 \\
2 & 1 & -1.9464 & -0.0514 & 1.0230 \\
2 & 2 & -2.5082 & -0.1765 & 1.0107 \\
2 & 3 & -3.0147 & -0.2858 & 1.0012 \\
\hline
\end{tabular}

chemical reaction, thermal radiation and heat generation for the improvement of the boundary layer flow has been taken into consideration. The effects of the various parameters $S, M, E c, Q, P r, R, S c$ and $C r$ on the fluid flow and temperature characteristics are discussed. From the acquired solution, the following results can be obtained:

- It has been concluded from the results that an increase in couple stress parameter $S$ enhance the flow velocity and reduce the temperature of the fluid.

- In the presence of magnetic field $M$, the fluid flow velocity is reduces and enhances the temperature profiles.

- The temperature of the couple stress fluid is increases due to increase in $\operatorname{Pr}, E c$ and $Q$ but decreases with $R$.

- The concentration of the couple stress fluid is reduces due to enhance in the chemical reaction parameter $\mathrm{Cr}$ and the Schmidt number $S c$.

\section{REFERENCES}

Alam, M., Rahman, M., and Sattar, M., 2008, "Effects of Chemical Reaction and Thermophoresis on Magneto-Hydrodynamic Mixed Convective Heat and Mass Transfer Flow Along an Inclined Plate in the Presence of Heat Generation and (Or) Absorption with Viscous Dissipation and Joule Heating," Canadian Journal of Physics, 86(9), 1057-1066. http://dx.doi.org/10.1139/P08-037.

Chang-Jian, C.W., and Chen, C.K., 2008, "Bifurcation Analysis of Flexible Rotor Supported by Couple-Stress Fluid Film Bearings with Non-Linear Suspension Systems," Tribology International, 41(5), 367-386.

http://dx.doi.org/10.1016/j.triboint.2007.09.007.

Ferdows, M., and M. Al-Mdallal, Q., 2013, "Effectsof Order of Chemical Reaction on a Boundary Layer Flow with Heat and Mass Transfer Over a Linearly Stretching Sheet," American Journal of Fluid Dynamics, 2(6), 89-94.

http://dx.doi.org/10.5923/j.ajfd.20120206.01.

Govardhan, K., Nagaraju, G., Kaladhar, K., and Balasiddulu, M., 2015, "MHD and Radiation Effects on Mixed Convection Unsteady Flow of Micropolar Fluid Over A Stretching Sheet," Procedia Computer Science, 57, 65-76.

http://dx.doi.org/10.1016/j.procs.2015.07.366.
Hayat, T., Mustafa, M., Iqbal, Z., and Alsaedi, A., 2013, "Stagnation-Point Flow of Couple Stress Fluid with Melting Heat Transfer," Applied Mathematics and Mechanics, 34(2), 167-176.

http://dx.doi.org/10.1007/s10483-013-1661-9.

Hussain, M., Ashraf, M., Nadeem, S., and Khan, M., 2013, "Radiation Effects on the Thermal Boundary Layer Flow of a Micropolar Fluid Towards a Permeable Stretching Sheet," Journal of the Franklin Institute, 350(1), 194-210. http://dx.doi.org/10.1016/j.jfranklin.2012.07.005.

Kameswaran, P.K., Narayana, M., Makanda, G., and Sibanda, P., 2012, “On Radiation Effects on Hydromagnetic Newtonian Liquid Flow Due to an Exponential Stretching Sheet," Boundary Value Problems, 1, 105.

http://dx.doi.org/10.1186/1687-2770-2012-105.

Khan, N.A., Riaz, F., and Khan, N.A., 2013, "Heat Transfer Analysis for Couple Stress Fluid over a Nonlinearly Stretching Sheet," Nonlinear Engineering, 2, 3-4. http://dx.doi.org/10.1515/nleng-2013-0014.

Khan, N.A., Riaz, F., and Sultan, F., 2014, "Effects of Chemical Reaction and Magnetic Field on a Couple Stress Fluid Over a Non-Linearly Stretching Sheet," The European Physical Journal Plus, 129(1), 18. http://dx.doi.org/10.1140/epjp/i2014-14018-2.

Patil, P., and Chamkha, A.J., 2013, "Heat and Mass Transfer from Mixed Convection Flow of Polar Fluid Along a Plate in Porous Media With Chemical Reaction," International Journal of Numerical Methods for Heat \& Fluid Flow, 23(5), 899926.

http://dx.doi.org/10.1108/HFF-03-2011-0060.

Patil, P., and Kulkarni, P., 2008, "Effects of Chemical Reaction on Free Convective Flow of a Polar Fluid Through a Porous Medium in the Presence of Internal Heat Generation," International Journal of Thermal Sciences, 47(8), 1043-1054. http://dx.doi.org/10.1016/j.ijthermalsci.2007.07.013.

Rahman, M.M., and Al-Lawatia, M., 2010, "Effects of Higher Order Chemical Reaction on Micropolar Fluid Flow on a Power Law Permeable Stretched Sheet With Variable Concentration in a Porous Medium," The Canadian Journal of Chemical Engineering, 88(1), 23-32. http://dx.doi.org/10.1002/cjce.20244.

Shaw, S., Kameswaran, P.K., and Sibanda, P., 2013, "HomogeneousHeterogeneous Reactions in Micropolar Fluid Flow From a Permeable Stretching or Shrinking Sheet in a Porous Medium," Boundary Value Problems, 1, 77. http://dx.doi.org/10.1186/1687-2770-2013-77.

Soundalgekar, V.M., 1971, "Effects of Couple Stresses in Fluids on Dispersion of a Solute in a Channel Flow," Physics of Fluids, 14(1), 19. http://dx.doi.org/10.1063/1.1693276.

Stokes, V.K., 1966, “Couple Stresses in Fluids,” Physics of Fluids, 9, 1709. http://dx.doi.org/10.1063/1.1761925.

Turkyilmazoglu, M., 2014, "Exact Solutions for Two-Dimensional Laminar Flow Over a Continuously Stretching or Shrinking Sheet in an Electrically Conducting Quiescent Couple Stress Fluid," International Journal of Heat and Mass Transfer, 72, 1-8. http://dx.doi.org/10.1016/j.ijheatmasstransfer.2014.01.009. 